\title{
Proposal of a Methodology for the Sustainability Assessment of Cryptocurrencies
}

\author{
Marco Näf \\ ZHAW \\ marco.naef@thurweb.ch
}

\author{
Thomas Keller \\ ZHAW \\ kell@zhaw.ch
}

\author{
Roger Seiler \\ ZHAW \\ seir@zhaw.ch
}

\begin{abstract}
As cryptocurrencies are becoming more and more widespread and their power consumption has caught the attention of the public, it seems worthwhile to investigate their effects on the environment, economy and society. In the scientific literature, a clear focus on the high power consumption of the market-dominating Bitcoin can be seen in the sustainability assessment of cryptocurrencies. In order to build a comprehensive understanding of cryptocurrencies' sustainability other aspects should be considered as well instead of narrowing down the scope of analysis to power consumption. Therefore, a holistic definition of sustainability in the context of cryptocurrencies is proposed. Building upon this definition a methodology for assessing a cryptocurrencies' sustainability is derived in this paper and subsequently applied to ten cryptocurrencies.
\end{abstract}

\section{Introduction}

The scientific investigation of the sustainability of cryptocurrencies is in its early stages. Most studies are available on Bitcoin's energy consumption [1]-[5], whereas other cryptocurrencies are rarely considered. Some studies focus on the comparison of consensus algorithms, using few or no quantifiable criteria or sustainability indicators [6]-[11]. Non-scientific quantitative comparisons mainly consider financial profitability and provide recommendations for investment decisions [12]-[14].

Currently, there is neither a clear definition of sustainability in connection with cryptocurrencies nor a generally accepted methodology for its investigation. The strong focus on Bitcoin has led to a generalization and neglects the fact that various cryptocurrencies are already traded on the market. A major research gap is the lack of a scientifically derived methodology with quantifiable criteria that allows to uniformly test and compare different cryptocurrencies for their sustainability. Furthermore, as mentioned in the abstract, most definitions and studies focus on a single quantitative criterion such as power consumption. This work proposes a mix of different quantitative as well as qualitative measures. The sustainability assessment of several cryptocurrencies can serve as a basis for a discussion on how to make existing or new digital currencies more sustainable.

\section{Objective}

The main goal of this work is to define sustainability and to derive criteria to determine the sustainability of cryptocurrencies. Combining both quantitative and qualitative criteria, a methodology shall be proposed that enables the structured assessment of a cryptocurrencies' sustainability. This allows the conscious selection of sustainable cryptocurrencies and the identification of product improvements potentials by means of specific indicators. In principle, the quantitative criteria would also allow an automated sustainability assessment.

\section{Methodology}

Since the goal of this work is the development of a methodology, relevant data was collected by means of literature study and qualitative research to achieve the study objective. For the literature analysis, categorical search terms were defined and applied in ten scientific literature databases. After the initial literature analysis, it was concluded that the scientific evidence on the topic were insufficient to meet the study objective. Therefore, in a second phase the gained insights were discussed with five blockchain experts by means of delphi method. Three of the interview partners are professors for blockchain and distributed ledger technology at Swiss universities. The other two offer consulting services for the enterprise use of blockchain technology, whereby one has an explicit focus on optimizing companies' positive impact. 


\section{Scope}

The Cambridge Centre for Alternative Finance conducted a study analyzing the regulation of cryptoassets in 23 jurisdictions. The classification of cryptotokens is essential for the various states to issue targeted regulations. $32 \%$ of the jurisdictions examined, have defined the following three categories of crypto-tokens [15]:

- Payment tokens are primarily used as digital means of payment or exchange. Cryptocurrencies are assigned to this category.

- Utility tokens are used for the use of platforms and decentralized applications. They have a usage value.

- Investment Tokens (Security Tokens) are assets such as shares, bonds or real estate. In theory, an investment token can be created for each asset [16]. Tokens deposited with real assets such as fiat currencies, gold or real estate are often referred to as stablecoins and are also assigned to this category [16].

Some crypto-tokens can be assigned to several categories, these are called hybrid tokens. However, often one category is predominant, e.g. Ethereum can be used as a payment token and utility token but is primarily designed as a utility token. Seldomly, cryptotokens cannot be assigned to any of the three categories.

The various types of crypto-tokens sometimes exhibit major differences in their objectives. This makes it impossible to develop and apply a uniform methodology for sustainability assessment. Therefore, we deliberately focus on payment tokens in this paper.

\section{Definition of sustainability}

The term "sustainability" has positive connotations, yet is also abstract and there is no uniform and clearly defined understanding as it is also used in the most diverse areas [17]. Multiple Perspectives must be considered when defining the term sustainability because cryptocurrencies are complex socio-economic systems in order to gain a broader understanding.

\subsection{The "classical" understanding of sustainability:}

Since its very first mention, the term sustainability has been associated with long-term thinking and the aim of ensuring lasting ecological as well as economic stability [18]. Probably the most well-known concept of sustainability emerged from the work of the Norwegian politician Gro Harlem Brundtland, who founded the World Commission on Environment and Development in Geneva in 1984: "Sustainable development is development that meets the needs of the present without compromising the ability of future generations to meet their own needs" [19]. In 1997, John Elkington presented the concept of the Triple Bottom Line, which is commonly used today. It is based on the Brundtland Report and the Rio Conference. Today it is often regarded as a synonym for sustainability [20] combining the dimensions of ecological responsibility, social justice and economic success as well as calling for a balanced consideration of all three dimensions.

\subsection{Sustainability in the ICT context}

In information and communication technology, various research fields around the concept of sustainability have emerged: Environmental Informatics, Computational Sustainability, Sustainable HCI, Green IT/ICT and ICT for Sustainability [21]. Software products such as cryptocurrencies are immaterial goods. Therefore, their effects on the physical world are of an indirect nature. They are not subject to wear and tear and can be copied without much effort and do not produce emissions when deleted. Hence, software seems to be a sustainable product. However, software products can differ considerably in terms of their impact on natural resources regardless of their functionality. This is especially true for cryptocurrencies. Two main drivers of the emissions are caused by the use of software [22]:

- The energy flow through the hardware on which the software runs.

- The flow of the hardware through the organizations that use it.

\subsection{State of research of sustainability and cryptocurrencies}

The authors identified 28 relevant studies on sustainability in the context of cryptocurrencies. These could be allocated to the following six categories:

Three-dimensional sustainability of blockchain and cryptocurrencies:

Studies in this category have attempted to present a holistic picture of the sustainability of cryptocurrencies. They include considerations of social, economic and ecological aspects.

Ethical aspects of distributed ledger systems and cryptocurrencies: Studies in this category have made ethical reflections on distributed transaction systems and cryptocurrencies on micro-, meso- and macro-level.

Energy consumption and CO2-emissions of cryptocurrencies: When measuring the energy consumption of cryptocurrencies, a clear focus on Bitcoin can be seen in the literature. In addition, there 
are several studies that assess energy consumption at the level of the consensus mechanism.

Value contribution of crypto-tokens to sustainable development: Studies assigned to this category show the potential of distributed ledger technology to contribute to sustainable development in sectors such as agriculture, state government, finance, energy or health care.

Governance of cryptocurrencies: Many cryptocurrencies are based on a decentralized, permissionless blockchain, which is characterized by its openness and the formal equality of the participants. However, this anarchic governance also poses many challenges that can threaten the long-term existence. Studies in this category consider the vulnerabilities to recentralization, informal coalitions of powerful actors, protocol change processes and incentives for mass collaboration.

Acceptance of distributed ledger systems and cryptocurrencies: To ensure the long-term existence of a cryptocurrency, it must be widely accepted by various stakeholders. Technology acceptance models for blockchain technology and cryptocurrencies have been developed in various scientific articles. Most of the models examine the factors that promote acceptance by end users. One study also examined the acceptance by developers.

\subsection{Sustainability requirements for crypto- currencies}

Reviewing the sustainability literature, the authors defined 78 requirements for sustainable cryptocurrencies. Subsequently, these requirements were clustered into 13 categories. These categories are interconnected and influence each other. For example, cryptocurrencies with a more centralized governance tend to consume less environmental resources. Hereafter, the 13 categories are described.

1. Value contribution to sustainable development: A cryptocurrency shall offer long-term economic, social and environmental value for various stakeholders. An imbalance of the three sustainability dimensions must be avoided. It should contribute to sustainable development solving a practical problem and does not remain a purely theoretical construct.

2. Efficient use of ecological resources: The cryptocurrency consumes as few resources as necessary to generate the added value it pursues. The administrators and network participants of the cryptocurrency are constantly refining it in order to reduce the energy consumption.

3. Long-term financial stability: The cryptocurrency should include mechanisms (on-chain and off-chain) that ensure long-term funding. The cryptocurrency is issued fairly and transparently from the beginning and there is a broad distribution of coins. The combination of a stable market position and low volatility protects stakeholders and promotes its use as a means of payment.

4. Technical maturity: The codebase of the cryptocurrency shall be in a mature stage, offer a high level of technical security and prevent the exploitation of security vulnerabilities. The cryptocurrency is regularly and comprehensively tested for technical errors and security gaps are quickly closed.

5. Technical performance: The cryptocurrency network shall be powerful and scalable. A high number of transactions can be processed in a short time with low fees.

6. Participative culture: Positive behavior of stakeholders who contribute value to the cryptocurrency network is encouraged and rewarded. There is an active ecosystem and an established sense of belonging and community, with an established value system providing guidance. The cryptocurrency is widely accepted and supported. Discrimination within the ecosystem is prevented and human rights as well as dignity are respected at all times.

7. Adaptability - Coordinated governance: Despite the decentralization, the cryptocurrency is coordinated and transparently managed. Many competent developers support the cryptocurrency and are encouraged to improve the cryptocurrency. The administration of the cryptocurrency is transparent and there is a coordinated innovation management, clear structures and processes. An established procedure for conflict resolution enables a constructive exchange within the network. The opinions of different, committed stakeholder groups are taken into account when making decisions.

8. Legal compliance: The cryptocurrency is in accordance with the law and there is cooperation with legislators, while respecting ethical aspects.

9. Trustworthiness of developers and administrators: The developers and administrators of a cryptocurrency are trustworthy. They are not involved in any illegal activities and support the continued existence of the cryptocurrency.

10. Knowledge transfer: The promotion of distributed ledger technology shall be supported by knowledge transfer. The source code of the cryptocurrency is publicly available, open source software is used and the development of industry standards is supported. Stakeholders have the opportunity to acquire knowledge about the cryptocurrency through concise documentation.

11. Network security: Network attacks are prevented by a high degree of decentralization and protective mechanisms. Potential attack areas have been 
identified and, if possible, solutions have been developed. Dependence on individuals, states, banks or technology companies is prevented. Confidence in network security is ensured at all times.

12. Protection of stakeholders: Incorrect application by stakeholders is prevented by clear operating and safety instructions. Stakeholders' privacy and data are protected, while the misuse of the cryptocurrency for criminal activities is prevented as far as possible.

13. Established infrastructure: There is a comprehensive infrastructure for easy and secure use of the cryptocurrency.

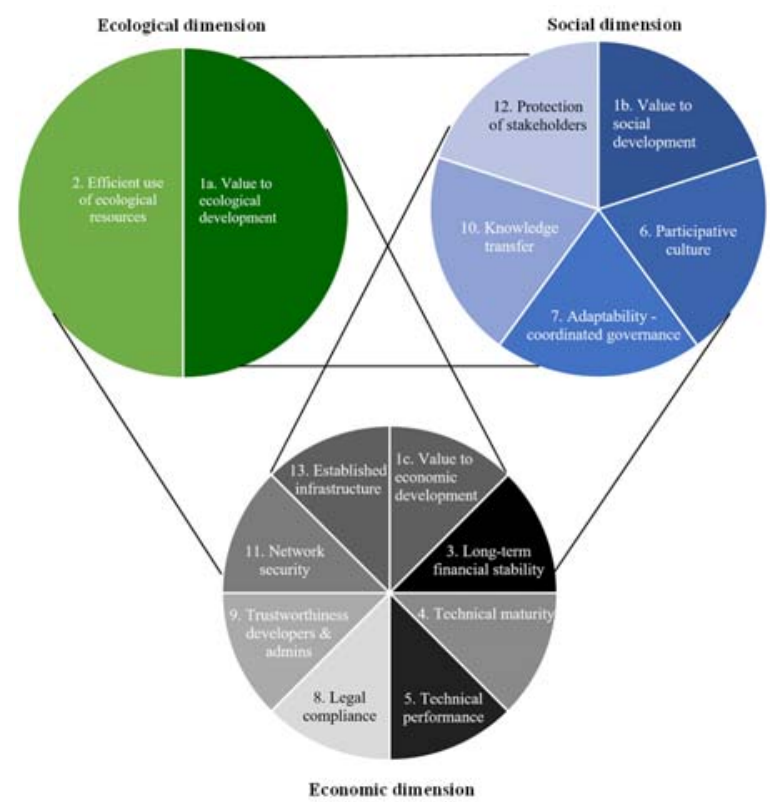

Figure 1. Sustainability requirement categories for cryptocurrencies

Connecting the requirement categories, the authors propose the following sustainability definition for cryptocurrencies:

"A sustainable cryptocurrency makes an economic, social and ecological contribution to sustainable development in the form of a scalable, decentralized and widely accepted payment system. As a socio-economic system, it involves independent people worldwide through clever mechanisms and procedures for longterm self-preservation and enables them to create added value through their participation. The protocol rules are clearly defined as well as communicated by the developers of the cryptocurrency from the very beginning and a well thought-out and transparent distribution of the units takes place. Despite the decentralization of the various actors, coordination is guaranteed. Through clearly defined and transparent processes, as well as taking into account the interests of different stakeholders, the cryptocurrency is continuously being enhanced. Central administrative bodies and intermediaries are avoided, whereby the trust of the various participants in the technology and the ecosystem is regarded as essential. Network security is maintained at all times. While energy consumption plays an important role in the first generation of cryptocurrencies for maintaining network consensus, solutions are currently emerging that are increasingly resource-efficient".

\subsection{Methodology of sustainability assessment}

The 13 sustainability categories and the sustainability definition form the basis for the development of the methodology for the sustainability assessment of cryptocurrencies. It enables end users to acquire knowledge about the sustainability of cryptocurrencies in a structured way and thus enables the selection of a cryptocurrency according to their subjective preferences. On the other hand, developers as well as administrators can use the methodology to check the sustainability of their cryptocurrency by means of concrete indicators and derive action measures to increase the sustainability of their product. For developers of new cryptocurrencies, the methodology offers a framework for orientation in order to develop a sustainable product.

In order to find suitable indicators for the sustainability assessment of cryptocurrencies, the authors have conducted a further literature review and derived indicators directly from the sustainability definition, too. By discussing the indicators with experts, further indicators could be identified and were included. The authors examined the suitability of an indicator for the sustainability assessment by means of an exclusion procedure with six criterias.

The authors decided to make a condensed version with 12 indicators and a detailed version with 42 indicators of the methodology. The short version allows a quick, first comparison of the cryptocurrencies for their sustainability. The short version includes only quantitative indicators to allow objective evaluations. The long version includes several sustainability aspects and draws a more detailed sustainability picture of a cryptocurrency. All quantitative indicators of the short version are retained in the long version. In addition, other quantitative indicators are also included to show additional aspects. However, it is not possible to record these additional quantitative indicators for all cryptocurrencies, which is why they were excluded in the short version. For example, the indicator "Hashrate in $\mathrm{TH} / \mathrm{s}$ " was not included in the short version because not all cryptocurrencies are mined. The qualitative indicators are prepared in the form of questions. This 
provides the users of the methodology with a questionnaire that allows them to check the most important sustainability aspects of a cryptocurrency in a structured way.

Before applying either the short or the long version of the methodology, it is recommended to collect the information according to Table 1 . The detailed version of the methodology is shown in Table 2. The dark gray highlighted indicators are the ones that are also used in the condensed version.

\section{Table 1 Recommended general information to be collected}

\begin{tabular}{|c|c|}
\hline Cryptocurrency name: & Website: \\
\hline \multicolumn{2}{|c|}{ Associated Organisations: Is there any organization, e.g. a foundation, which plays an important role in the cryptocurrency ecosystem? } \\
\hline \multicolumn{2}{|c|}{ Used DLT-Architecture: Blockchain, directed acyclic graph, ... } \\
\hline Consensus protocol: Proof of work, proof of stake, ... & Hash algorithm: SHA256, Scrypt, RandomX, ... \\
\hline $\begin{array}{l}\text { Max supply: Is there a max supply? If so, how many coins can be created } \\
\text { or have already been created? }\end{array}$ & $\begin{array}{l}\text { Circulating supply): How many coins have already been issued and are in } \\
\text { circulation? }\end{array}$ \\
\hline \multicolumn{2}{|c|}{$\begin{array}{l}\text { Source code information: } \\
\text { Is the source code publicly available, for example on GitHub? } \\
\text { Is the source code of the examined cryptocurrency based on the protocol of another cryptocurrency? }\end{array}$} \\
\hline \multicolumn{2}{|c|}{$\begin{array}{l}\text { Cryptocurrency creation: } \\
\text { There are three main mechanisms for creating cryptocurrencies [15]. } \\
\text { - Pre-mine: An instance creates all units in a batch as a single event; } \\
\text { - Continuous mining: Special network nodes called record producers ("miner", "staker", "baker", etc.) continuously create new units according to a } \\
\text { transparent, pre-determined procedure governed by the protocol. } \\
\text { - Hybrid: Some instances mine a certain proportion of the total final supply; the remaining units are then created by continuous mining. }\end{array}$} \\
\hline \multicolumn{2}{|c|}{$\begin{array}{l}\text { Network participation: Distributed ledger systems can be divided into public and private networks and in permissionless and permissioned networks } \\
\text { [23]. }\end{array}$} \\
\hline $\begin{array}{l}\text { Public \& permissionless: Within these systems the protocol can be } \\
\text { downloaded by anyone. Anyone can join the network and validate } \\
\text { transactions. }\end{array}$ & $\begin{array}{l}\text { Public \& permissioned: Within these systems the protocol can be } \\
\text { downloaded by anyone. However, only selected participants are allowed to } \\
\text { validate transactions. }\end{array}$ \\
\hline $\begin{array}{l}\text { Private \& permissionless: Only selected participants may join the } \\
\text { network, but all of them may validate transactions. }\end{array}$ & $\begin{array}{l}\text { Private \& permissioned: Only selected participants may join the network } \\
\text { and only selected participants are allowed to validate transactions. }\end{array}$ \\
\hline $\begin{array}{l}\text { Anonymity: } \\
\text { - address of the transaction sender: public or anonymous [24] } \\
\text { - address of the transaction recipient: public or anonymous [24] } \\
\text { - address list: public or anonymous [24] }\end{array}$ & $\begin{array}{l}\text { k between transaction sender and receiver: public or anonymous [24] } \\
\text { ansaction amount, data: public or anonymous [24] }\end{array}$ \\
\hline
\end{tabular}

Table 2 Methodology of sustainability assessment

\begin{tabular}{|c|c|}
\hline \multicolumn{2}{|r|}{ 1.Value contribution } \\
\hline \multicolumn{2}{|c|}{ Qualitative indicator } \\
\hline 1 & $\begin{array}{l}\text { Added value and purpose: What are the unique selling propositions of the cryptocurrency? Does the cryptocurrency contribute to sustainable } \\
\text { development? }\end{array}$ \\
\hline \multicolumn{2}{|r|}{ 2. Efficient use of ecological resources } \\
\hline \multicolumn{2}{|c|}{ Quantitative indicators } \\
\hline 2 & Total network energy consumption, during defined time period \\
\hline 3 & Ø Energy consumption per transaction, during defined time period \\
\hline \multicolumn{2}{|r|}{ Qualitative indicators } \\
\hline 4 & Efforts to reduce energy consumption: Are there efforts by developers/administrators to make the cryptocurrency more sustainable? \\
\hline 5 & Potential for ecological awareness: Are stakeholders made aware of a resource-saving usage of the cryptocurrency, e.g. on the project website? \\
\hline 6 & Required hardware: Which hardware is required to operate the different types of network nodes? \\
\hline \multicolumn{2}{|r|}{ 3. Long-term financial stability } \\
\hline \multicolumn{2}{|c|}{ Quantitative indicators } \\
\hline 7 & Market capitalization, market rank, market dominance in \%, reporting date \\
\hline 8 & Volatility, during defined time period \\
\hline 9 & Transaction volume, during defined time period \\
\hline 9.1 & - Ø Transaction volume per day in \$ \\
\hline 9.2 & $-\varnothing$ Number of transactions per day \\
\hline 10 & Coin distribution: Shares of the top 10 , top 100 , top 1,000 and top 10,000 addresses of the circulating supply, reference date \\
\hline \multicolumn{2}{|r|}{ Qualitative indicators } \\
\hline 11 & $\begin{array}{l}\text { Generation of the cryptocurrency: Was the generation of the cryptocurrency transparent and error-free? } \\
\text { (Different types of token generation: } 1 \text {. pre-mine, 2. continuous mining, 3. hybrid) }\end{array}$ \\
\hline 12 & $\begin{array}{l}\text { Initial distribution of the cryptocurrency: Was the initial distribution of the cryptocurrency transparent and error-free? } \\
\text { (Different types of initial distributions: } 1 \text {. pre-token-sale, 2. token-sale/ICO, 3. mining, 4. airdrop, 5. fork) }\end{array}$ \\
\hline 13 & $\begin{array}{l}\text { Distribution mechanism after initial distribution: How are the coins of the cryptocurrency distributed after the initial issuance or how is the supply } \\
\text { performed? How are the functionality and financing of the cryptocurrency (also after all coins are issued) ensured? }\end{array}$ \\
\hline \multicolumn{2}{|r|}{ 4. Technical maturity } \\
\hline \multicolumn{2}{|r|}{ Quantitative indicator } \\
\hline 14 & Foundation year (number of years in the market), reference date \\
\hline Qua & ative indicators \\
\hline
\end{tabular}


15 Project stage: 1) Concept stage, 2) Development, 3) Deployment, 4) Maintenance and further development

16 Technical maturity of the protocol: Are there or were there technical weaknesses on protocol level? How severe were or are these vulnerabilities? How fast have security vulnerabilities been closed in the past? Is there a bug bounty program and/or security audit?

Quantitative indicators

5. Technical performance

17 Confirmation latency, reference date

18 Transactions per second [TPS]

18.1 - Verified max TPS since foundation, reference date

18.2 - Theoretically possible TPS, reference date

19 Transaction costs in $\$$

19.1 - Average, during defined time period

19.2 - Median, during defined time period

\section{Quantitative indicators}

6. Participative culture

20 Activity in social networks, reference date

20.1 - Reddit: subscribers

20.2 - Reddit: $\varnothing$ active account

20.3 - Reddit: $\varnothing$ hot posts p.h.

20.4 - Reddit: $\varnothing$ new comments p.h.

20.5 - Facebook: likes

20.6 - Twitter: followers

21 Development activity, reference date

Specification Github client and main repository (MR)

21.1 - Github MR contributors

21.2 - Github MR commits since foundation - considering potential fork code

- Github MR commits 1y

Qualitative indicators

22 Participation incentives: What are the incentive mechanisms for different stakeholders to participate in the cryptocurrency ecosystem? This

indicator includes the identification of the relevant stakeholders.

23 Is there a code of ethics and/or conduct for the stakeholders?

\section{Adaptability - coordinated governance}

Qualitative indicators

24 Protocol changes: Is there a structured transparent process for proposing protocol changes? Who can propose changes? Who may decide on protocol change proposals? Who implements the protocol changes? Can the history of protocol changes be inspected?

25 Adherence to project roadmap: Who defines the project roadmap? Could the set goals be met in the past?

\section{Qualitative indicator}

8. Legal compliance

26 Accountability: Is there an organization or individuals who take responsibility for the cryptocurrency?

27 Cooperation with regulatory authorities: Is there a cooperative behavior towards regulators?

Qualitative indicator

9. Trustworthiness of developers / administrators

28 Who are the developers and administrators of the cryptocurrency? Do they have a track record? Were they involved in criminal activities?

\section{Qualitative indicators}

10. Knowledge transfer

29 Quality of the explanatory material: How easy is it for the various stakeholders to acquire knowledge of the cryptocurrency? Are there detailed

instructions, e.g. for the installation of node software?

30 Open Source: Is any software used open source?

Quantitative indicators

\section{Network security}

31 Network size: number of full nodes, reference date

32 Ø Hashrate considering the required hardware, Reference date (This indicator is only applicable to mined cryptocurrencies)

Qualitative indicators

33 Geographic distribution of full nodes

34 Geographic distribution of hashrate (This indicator is only applicable to mined cryptocurrencies)

35 Independence from organizations or individuals: Is there a dependency on organizations, mining pools, partners, individual key persons, etc.? Can a central authority significantly influence the network?

36 Network vulnerability resistance: To what kind of attacks is the cryptocurrency vulnerable to? What protection mechanisms exist to resist these attacks?

37 Number of incidents on network level: Have serious network security breaches occurred in the past?

38 Assessment of the technical maturity and diffusion of the consensus protocol and hash algorithm

Qualitative indicators

12. Protection of stakeholders

39 Have there been serious incidents in the ecosystem in the past, e.g. crypto exchanges, wallet solutions, etc.?

40 Are there any security instructions for using the cryptocurrency?

41 Potential for criminal abuse: How suitable is the cryptocurrency to be used for criminal activities, e.g. in darknet?

Qualitative indicator

\section{Established infrastructure}

42 Are there many services for the application of the cryptocurrency? Assessment of the number of acceptance points, wallets, crypto exchanges and

42 other services for using the cryptocurrency. 
Hereafter, the indicators are described that are included in the short version.

Total network energy consumption (2):

When assessing the power consumption of cryptocurrencies, the consensus protocol must be understood as a priority. Proof of Work (PoW) is at the moment the most commonly used mechanism for cryptocurrencies. In the literature, a distinction is made between two procedures for assessing the energy consumption of cryptocurrencies that use PoW: the economic top-down approach and the techno-economic bottom-up approach [25]. The underlying assumption within the top-down-approach is that miners' revenues and costs are related. The higher the income from mining, the more energy-hungry mining hardware can be operated [26]. The underlying idea of the bottom-up approach is that the hashrate of a network multiplied by the energy efficiency of the mining hardware and the energy efficiency of the data centers (cooling, supporting IT hardware, etc.) results in the power consumption of the cryptocurrency network [27].

Energy consumption per transaction (3): The total power consumption is divided by the processed transactions in the same time period. However, this indicator has some limitations. The transaction throughput is only conditionally dependent on electricity consumption. For example, the adding of multiple mining servers increases power consumption, but must have no no effect on the number of transactions processed [25].

Market capitalization (7): The market capitalization defines the current price of a coin multiplied by the circulating supply. Market capitalization is an indicator for the level of investment risk [28].

Volatility (8): As a result of high price stability or a low volatility respectively, a cryptocurrency is more suited as a store of value and means of payment.

Transaction volume (9): This indicator allows to assess the effective use of the cryptocurrency [29], [30].

Foundation year (14): It is challenging to assess the technical maturity of a cryptocurrency by evaluating individual technical features, e.g. the fault-tolerance or collision resistance of the used hash-algorithm. Rather, the source code should be checked for errors, which is time-consuming. An indication for technical maturity is the duration, a cryptocurrency is available on the market as over the years, the resistance of the cryptocurrency to various attack patterns and faulty programming is revealed.

Confirmation latency (17): The confirmation latency is the minimal time until sufficient transactions are added to the distributed ledger so that the probability of retroactive manipulation of a previously added block or transaction is below a certain threshold [9]. If cryptocurrencies are to compete directly with fiat payment services, transaction speeds must be able to keep pace with their fiat competitors, at least to some extent.

Transactions per second (18): This indicator is associated with the scalability of a network [9]. The expandability of a distributed transaction system is limited by the number of transactions per second. In overloaded systems, transaction fees are used to prioritize transactions [16].

Transaction costs (19): Transaction fees are the difference between the amount sent and received in a transaction [9]. The median and mean value should be calculated.

Activity in social networks (20): This indicator can provide information about how many people are interested in a cryptocurrency and support it.

Development activity (21): Due to a constant development of the cryptocurrency, the longevity of the cryptocurrency is more likely to be guaranteed. To measure the development activity of a cryptocurrency, the researchers Gräbe et al. (2020) recommend assessing the number of people who participate in the development of a cryptocurrency [31]. The source codes of cryptocurrencies are usually publicly available. With the help of the indicator GitHub Commits of the Main Repository [MR] the frequency of code updates can be measured. When assessing MR commits, it must be taken into account that cryptocurrencies have different numbers of repositories and therefore only a fraction of the development activity is assessed with this indicator.

Network size (31): Network nodes are computers that are connected to a cryptocurrency network and use the P2P-protocol, which allows them to communicate and process transaction information within the network [32]. For each cryptocurrency, the developers specify the types of network nodes that are intended to be used, which determines the possibilities for participation in the network. Most often, a distinction is made between two types of network nodes: full nodes and lightweight nodes [16]. For the assessment, full nodes and their counterparts in other systems are particularly relevant, since they are used to realize distributed data storage. The more full nodes are active in a network, the more robust and resilient it is [16].

\section{Illustrative use of the short version}

In this work, the short version is applied to the cryptocurrencies Bitcoin, XRP, Bitcoin Cash, Litecoin, Stellar Lumens, Monero, Dash, Zcash, Decred and Nano to verify the practical suitability of the methodology. In order to be able to put the quantitative results obtained 
into a framework, the payment service providers VISA Inc. and PayPal Holdings Inc. will also be examined using the same methodology. Table 3 shows only the comparison between Visa, Bitcoin and Bitcoin Cash. The complete comparison including visualizations is available online ${ }^{1}$.

Table 3. Illustrative use of the short version

\begin{tabular}{|c|c|c|c|c|}
\hline \# & Indicator & $\begin{array}{l}\text { Visa Inc. } \\
\text { (V) }\end{array}$ & $\begin{array}{l}\text { Bitcoin } \\
\text { (BTC) }\end{array}$ & $\begin{array}{l}\text { Bitcoin } \\
\text { Cash }(\mathrm{BCH})\end{array}$ \\
\hline 1 & \multicolumn{4}{|c|}{ Energy consumption } \\
\hline & $\begin{array}{l}\text { Annual total network } \\
\text { energy consumption }\end{array}$ & $\begin{array}{l}0.197 \mathrm{TWh} \\
\quad(2018)\end{array}$ & - & - \\
\hline & $\begin{array}{l}\text { - Top-down-method, } \\
01.01 .-01.03 .20, \\
\text { projected to one year }\end{array}$ & - & $\begin{array}{c}74.286 \\
\text { TWh }\end{array}$ & $2.815 \mathrm{TWh}$ \\
\hline & $\begin{array}{l}\text { - Bottom-up-method, } \\
\text { best guess (BU-BG), } \\
01.01 .-01.03 .20, \\
\text { projected to one year }\end{array}$ & - & $79.68 \mathrm{TWh}$ & $3.035 \mathrm{TWh}$ \\
\hline \multirow[t]{3}{*}{2} & \multicolumn{4}{|c|}{ Energy consumption per transaction } \\
\hline & $\begin{array}{l}\text { Ø Energy } \\
\text { consumption per } \\
\text { transaction, } 01.01 \text {. - } \\
01.03 .2020(\mathrm{BU}- \\
\mathrm{BG})\end{array}$ & $\begin{array}{l}0.0016 \\
\mathrm{kWh} \\
(2018)\end{array}$ & $\begin{array}{c}676.7 \mathrm{kWh} \\
(3.734 \\
\text { TPS })\end{array}$ & $\begin{array}{l}183.72 \mathrm{kWh} \\
(0.524 \mathrm{TPS})\end{array}$ \\
\hline & $\begin{array}{l}\text { Ø Energy } \\
\text { consumption per } \\
\text { transaction, with } \\
220.752 \text { mio. } \\
\text { transactions per year } \\
\text { - hypothetical max } \\
\text { BTC, 01.01. - } \\
01.03 .2020 \text { (BU- } \\
\text { BG) }\end{array}$ & $\begin{array}{c}0.895 \mathrm{kWh} \\
(2018,7 \\
\text { TPS })\end{array}$ & $\begin{array}{c}360.95 \\
\mathrm{kWh} \\
(7 \mathrm{TPS})\end{array}$ & $\begin{array}{c}13.75 \mathrm{kWh} \\
(7 \mathrm{TPS})\end{array}$ \\
\hline 3 & $\begin{array}{l}\text { Market } \\
\text { capitalization, } \\
\text { market rank, } \\
\text { market dominance } \\
\text { in \%, 16.03.20 }\end{array}$ & 295 bn. $\$$ & $\begin{array}{c}92.12 \mathrm{bn} . \\
\$ ., \# 1 \\
64.85 \%\end{array}$ & $\begin{array}{c}3.12 \mathrm{bn} . \$ \\
\# 5 \\
2.18 \%\end{array}$ \\
\hline 4 & $\begin{array}{l}\text { Volatility, 13.03.19 } \\
-13.03 .20\end{array}$ & $22.98 \%$ & $86.11 \%$ & $122.21 \%$ \\
\hline \multirow[t]{3}{*}{5} & \multicolumn{4}{|l|}{ Transaction volume } \\
\hline & $\begin{array}{l}\varnothing \text { transaction } \\
\text { volume in } \$ \text { per day, } \\
01.01 .-01.03 .2020\end{array}$ & $\begin{array}{l}8^{\prime} 619 \mathrm{bn} . \$ \\
(2019)\end{array}$ & $1.935 \mathrm{bn} . \$$ & $\begin{array}{c}186.485 \mathrm{~m} \\
\$\end{array}$ \\
\hline & $\begin{array}{l}\emptyset \text { number of } \\
\text { transactions per day, } \\
01.01 .-01.03 .2020\end{array}$ & $\begin{array}{l}378.984 \mathrm{~m} . \\
(2019)\end{array}$ & $317 ' 306$ & $44 ' 517$ \\
\hline 6 & $\begin{array}{l}\text { Maturity: } \\
\text { Foundation year; } \\
\text { number of years } \\
\text { since foundation }\end{array}$ & $1976(44)$ & $2009(11)$ & $2017(3)$ \\
\hline 7 & $\begin{array}{l}\text { Confirmation } \\
\text { latency } \\
\text { (non-scientific } \\
\text { literature) }\end{array}$ & $\begin{array}{l}\text { within a } \\
\text { few } \\
\text { seconds }\end{array}$ & $\begin{array}{l}10 \mathrm{~min} \text {; it is } \\
\text { recommend } \\
\text {-ed to wait } \\
6 \\
\text { transaction } \\
\mathrm{s}=60 \mathrm{~min}\end{array}$ & $\begin{array}{l}10 \mathrm{~min} \text {; it is } \\
\text { recommend } \\
\text {-ed to wait } \\
\quad 6 \\
\text { transactions } \\
=60 \mathrm{~min} \\
\end{array}$ \\
\hline 8 & \multicolumn{4}{|c|}{ Transaction per second (29) } \\
\hline & $\begin{array}{l}\text { Max TPS, during } \\
\text { one day, } 1 \mathrm{~J} . \text {, } \\
13.03 .20\end{array}$ & $\begin{array}{c}\varnothing 4^{\prime} 385 \\
\text { TPS (2019) }\end{array}$ & 5.25 & 7.64 \\
\hline & $\begin{array}{l}\text { Max TPS, during } \\
\text { one day, since } \\
\text { foundation }\end{array}$ & - & 5.76 & 25.1 \\
\hline
\end{tabular}

${ }^{1}$ https://drive.switch.ch/index.php/s/PVrFBy2zvHcUntW

\begin{tabular}{|c|c|c|c|c|}
\hline & $\begin{array}{l}\text { Estimated TPS } \\
\text { according to non- } \\
\text { scientific literature }\end{array}$ & $500^{\prime} 000$ & 7 & 250 \\
\hline 9 & \multicolumn{4}{|c|}{ Transaction costs in \$ (30) } \\
\hline & $\begin{array}{l}\text { Average, } 13.03 .19 \text { - } \\
13.03 .20\end{array}$ & $\begin{array}{c}0.0564 \$ \\
(2019)\end{array}$ & $0.64 \$$ & $0.005 \$$ \\
\hline & $\begin{array}{l}\text { Median, 13.03.19 - } \\
13.03 .20\end{array}$ & no data & $0.28 \$$ & $0.001 \$$ \\
\hline 10 & \multicolumn{4}{|c|}{ Activity in social networks } \\
\hline & $\begin{array}{l}\text { - Reddit: Subscribers } \\
(15.03 .20)\end{array}$ & $\begin{array}{l}\text { no Reddit } \\
\text { channel }\end{array}$ & $\begin{array}{l}\text { r/Bitcoin: } \\
\text { 1'318'182 }\end{array}$ & $\begin{array}{c}\text { r/Bitcoin- } \\
\text { cash: } \\
\text { 49'443; } \\
\text { r/btc: } \\
291^{\prime} 626 \\
\end{array}$ \\
\hline & $\begin{array}{l}\text { - Facebook: Likes } \\
(15.03 .20)\end{array}$ & $22^{\prime} 949^{\prime} 415$ & $518^{\prime} 990$ & $21^{\prime} 172$ \\
\hline & $\begin{array}{l}\text { - Twitter: Followers } \\
(15.03 .20)\end{array}$ & $\begin{array}{l}\text { @Visa: } \\
\text { 380K }\end{array}$ & $\begin{array}{l}\text { @ Bitcoin: } \\
\text { 1Mio. }\end{array}$ & $\begin{array}{l}\text { @ BitcoinCa } \\
\text { shA: } 12 \mathrm{~K}, \\
@ \text { Bitcoin } \\
\text { ABC: } 6 \mathrm{~K} \\
\end{array}$ \\
\hline 11 & \multicolumn{4}{|l|}{ Development activity } \\
\hline & $\begin{array}{l}\text { - Github } \\
\text { Contributors MR } \\
(16.03 .20) \\
\end{array}$ & N/A & $\begin{array}{l}\text { bitcoin: } \\
688\end{array}$ & $\begin{array}{l}\text { bitcoin-abc: } \\
526\end{array}$ \\
\hline & $\begin{array}{l}\text { - Github MR } \\
\text { commits since } \\
\text { foundation } \\
(16.03 .20)\end{array}$ & $\mathrm{N} / \mathrm{A}$ & $23 ' 179$ & $\begin{array}{l}17^{\prime} 939 \\
\text { (incl. fork- } \\
\text { code BTC) }\end{array}$ \\
\hline & $\begin{array}{l}\text { - Github MR } \\
\text { commits (1J., } \\
16.03 .20)\end{array}$ & N/A & $1^{\prime} 683$ & 1'346 \\
\hline 12 & $\begin{array}{l}\text { Number of full } \\
\text { nodes, } 14.03 .20 \text { (54) }\end{array}$ & N/A & $10 ' 365$ & $1 ' 591$ \\
\hline
\end{tabular}

Based on the collected data, the authors created sustainability profiles for the cryptocurrencies. These allow quick conclusions about the strengths and weaknesses of these. For the creation of the sustainability profiles, the authors defined individual scaling per dimension. For most dimensions, logarithmic scaling was used, since there were partly substantial value differences between the cryptocurrencies. Two examples of these sustainability profiles are presented in Figure 2 and Figure 3.

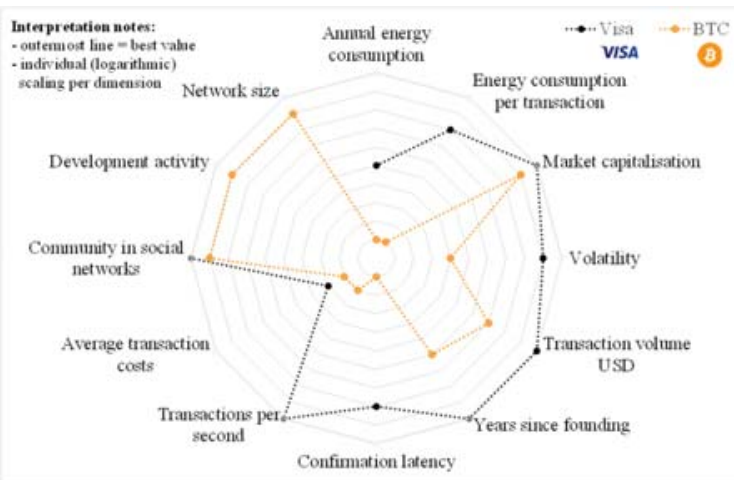

Figure 2: Sustainability profiles Visa and Bitcoin 


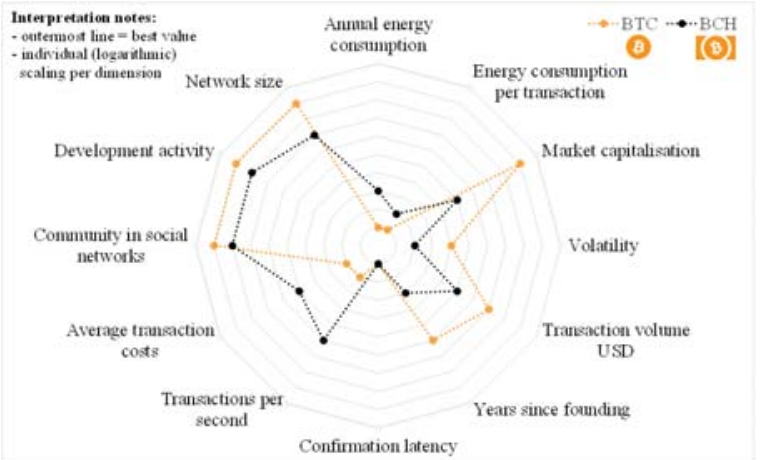

Figure 3: Sustainability profiles Bitcoin Cash and Bitcoin

\section{Conclusions}

The proposed methodology was elaborated considering transparency, relevance, comparability, scalability and fairness. Most but not all decisions along the way to the final methodology are traceable back to literature or are logically derived. All the same the identified indicators grouped into categories and dimensions can be easily challenged and may be perceived as partially arbitrary. Only the application in practice may give a hint if the methodology leads to more insight and leads to better decisions regarding the use of cryptocurrencies. At this point of time one can only speculate.

Apart from this fundamental problem a key success factor is the measurement of the quantitative indicators. Some of the indicators are best guesses, some can only be measured by insiders and must therefore be considered with care. An improvement would be the automized measurement of as many of the indicators as possible.

Another challenge are the qualitative indicators. They always come with a subjective part. The delphi method may be a good approach to come to a common understanding. This would however involve a group of experts with a good understanding of the subject matter.

The methodology is grounded on a definition of sustainability. This definition is anchored itself in definitions of sustainability of three different fields. The chosen wording of sustainability for cryptocurrencies reflects the requirements from these three fields. This derivation can be challenged. As with many argumentations one can weigh arguments differently and will therefore come to different solutions and hypotheses. Therefore, the proposed definition of sustainability is our best effort and we are looking forward for constructive feedback.
Only an application in practice will eventually show the pragmatism and the accuracy of the methodology.

\section{References}

[1] C. Stoll, L. Klaaßen, and U. Gallersdörfer, "The Carbon Footprint of Bitcoin," Joule, vol. 3, no. 7, pp. 1647-1661, Jul. 2019, doi: 10.1016/j.joule.2019.05.012.

[2] M. J. Krause and T. Tolaymat, "Quantification of energy and carbon costs for mining cryptocurrencies," Nat. Sustain., vol. 1, no. 11, pp. 711-718, Nov. 2018, doi: 10.1038/s41893-018-0152-7.

[3] H. McCook, "The Cost \& Sustainability of Bitcoin," 2018. Accessed: Dec. 12, 2019. [Online]. Available: https://www.academia.edu/37178295/The_Cost_and_S ustainability_of_Bitcoin_August_2018_.

[4] A. de Vries, "Bitcoin's Growing Energy Problem," Joule, vol. 2, no. 5, pp. 801-805, May 2018, doi: 10.1016/j.joule.2018.04.016.

[5] H. Vranken, "Sustainability of bitcoin and blockchains," Curr. Opin. Environ. Sustain., vol. 28, pp. 1-9, Oct. 2017, doi: 10.1016/j.cosust.2017.04.011.

[6] S. S. Hazari and Q. H. Mahmoud, "Comparative evaluation of consensus mechanisms in cryptocurrencies," Internet Technol. Lett., vol. 2, no. 3, p. e100, May 2019, doi: 10.1002/it12.100.

[7] S. Zhang and J.-H. Lee, "Analysis of the main consensus protocols of blockchain," ICT Express, p. S240595951930164X, Aug. 2019, doi: 10.1016/j.icte.2019.08.001.

[8] L. M. Bach, B. Mihaljevic, and M. Zagar, "Comparative analysis of blockchain consensus algorithms," in 2018 41st International Convention on Information and Communication Technology, Electronics and Microelectronics (MIPRO), Opatija, May 2018, pp. 1545-1550, doi: 10.23919/MIPRO.2018.8400278.

[9] N. Chaudhry and M. M. Yousaf, "Consensus Algorithms in Blockchain: Comparative Analysis, Challenges and Opportunities," 2018, Accessed: Nov. 18, 2019. [Online]. Available: https://ieeexplore.ieee.org/servlet/opac?punumber $=8624$ 241.

[10] M. Garriga, M. Arias, and A. De Renzis, "Blockchain and Cryptocurrency: A comparative framework of the main Architectural Drivers," ArXiv181208806 Cs, Nov. 2018, Accessed: Jul. 09, 2020. [Online]. Available: http://arxiv.org/abs/1812.08806.

[11] K. Wagner, T. Keller, and R. Seiler, "A comparative analysis of cryptocurrency consensus algorithms," 2019, pp. 217-225, Accessed: Jul. 10, 2020. [Online]. Available: https://digitalcollection.zhaw.ch/handle/11475/18789.

[12] weisscrypto.com, "Weiss Crypto Ratings." https://weisscrypto.com/en/coins (accessed Feb. 09, 2020).

[13] flipsidecrypto.com, "Ratings | Flipside Crypto." https://flipsidecrypto.com/products/ratings (accessed Feb. 09, 2020).

[14] tokeninsight.com, "Cryptocurrency rating_Blockchain rating_Project rating." 
https://tokeninsight.com/rating?type=1 (accessed Feb. 09, 2020).

[15] A. Blandin et al., "Global Cryptoasset Regulatory Landscape Study," SSRN Electron. J., 2019, doi: 10.2139/ssrn.3379219.

[16] P. Egloff and E. Turnes, Blockchain für die Praxis Kryptowährungen, Smart Contracts, ICOs und Tokens. 2019.

[17] I. Pufé, Nachhaltigkeit, 3., überarbeitete und erweiterte Auflage. Konstanz München: UVK Verlagsgesellschaft $\mathrm{mbH}$ mit UVK/Lucius, 2017.

[18] F. Ekardt, Theorie der Nachhaltigkeit: Ethische, rechtliche, politische und transformative Zugänge - am Beispiel von Klimawandel, Ressourcenknappheit und Welthandel. Nomos Verlagsgesellschaft GmbH \& Co. KG, 2016.

[19] World Commission on Environment and Development, Ed., Our common future. Oxford; New York: Oxford University Press, 1987.

[20] J. Elkington, Cannibals with forks: the triple bottom line of 21st century business, Reprint. Oxford: Capstone, 2002.

[21] ICT4S, ICT innovations for sustainability. Cham Heidelberg New York Dordrecht London: Springer, 2015.

[22] E. Kern et al., "Sustainable software products—-Towards assessment criteria for resource and energy efficiency," Future Gener. Comput. Syst., vol. 86, pp. 199-210, Sep. 2018, doi: 10.1016/j.future.2018.02.044.

[23] M. Rauchs et al., "Distributed Ledger Technology Systems: A Conceptual Framework," SSRN Electron. J., 2018, doi: 10.2139/ssrn.3230013.

[24] Y. Sun and Y. Zhang, "Privacy in Cryptocurrencies: An Overview.” https://medium.com/@yi.sun/privacy-in- cryptocurrencies-d4b268157f6c (accessed Apr. 11, 2020).

[25] Cambridge Centre for Alternative Finance, "Cambridge Bitcoin Electricity Consumption Index (CBECI)," https://cbeci.org. https://cbeci.org/methodology/ (accessed Feb. 22, 2020).

[26] J. Koomey, "Estimating Bitcoin Electricity Use: A Beginner's Guide, Coin Center Report," 2019. Accessed: Mar. 19, 2020. [Online]. Available: https://coincenter.org/entry/bitcoin-electricity.

[27] M. Bevand, "Electricity consumption of Bitcoin: a market-based and technical analysis," Mar. 10, 2017. http://blog.zorinaq.com/bitcoin-electricity-consumption/ (accessed Mar. 06, 2020).

[28] J. Koenraadt and E. Leung, "The Impact of Regulation and Transparency in the Cryptocurrency Market," SSRN Electron. J., 2019, doi: 10.2139/ssrn.3339197.

[29] M. Dorofeyev, M. Kosov, V. Ponkratov, A. Masterov, A. Karaev, and M. Vasyunina, "Trends and prospects for the development of blockchain and cryptocurrencies in the digital economy," Eur. Res. Stud. J., vol. 21, pp. 429 445,2018

[30] A. Glarner et al., "Conceptual Framework for Legal and Risk Assessment of Crypto Token.” May 01, 2018, [Online]. Available: https://www.mme.ch/fileadmin/files/documents/180501 _BCP_Framework_for_Assessment_of_Crypto_Tokens - Block 2.pdf.

[31] F. Gräbe, N. Kannengiesser, S. Lins, and A. Sunyaev, "Do Not Be Fooled: Toward a Holistic Comparison of Distributed Ledger Technology Designs," 2020, doi: 10.24251/HICSS.2020.770.

[32] BitcoinWiki, "Crypto Node," May 05, 2020. https://en.bitcoinwiki.org/wiki/Node (accessed May 05, 2020) 